\title{
Le radon 222 et ses descendants à vie courte dans l'environnement atmosphé- rique : origine et méthodes de mesure*
}

\author{
J. CHARUAU 1 , V. LABED ${ }^{1}$, M.C. ROBÉ1, J.C. THÉVENIN ${ }^{1}$, C. AUBERT ${ }^{2}$, \\ J. FAZILEABASSE ${ }^{3}$, D. FLORENCE ${ }^{4}$, C. GIBAUD ${ }^{5}$, H. HELESCHEWITZ ${ }^{6}$, \\ D. KLEIN 7 , N. LEMAITRE6 ${ }^{6}$, G. TYMEN 8
}

(Manuscrit reçu le 2 août 1995, accepté le 28 avril 1996)

\begin{abstract}
RÉSUMÉ Pour l'homme, le radon est la source permanente principale d'exposition naturelle aux rayonnements ionisants. Ce document synthétise les connaissances générales sur l'origine du radon 222 et son évolution dans différents milieux atmosphériques. Il présente diverses méthodes de mesure de l'activité volumique radon et de la concentration. en énergie alpha potentielle de ses descendants à vie courte. Il a été élaboré dans le cadre de la commission M60-3 du Bureau de Normalisation d'Équipements Nucléaires (BNEN) qui dépend de l'Association Française de Normalisation (AFNOR).
\end{abstract}

ABSTRACT Radon is the main source of man's exposure to natural ionizing radiation. This document summarizes the general knowledge of the origin of radon 222 and its development in various air environnements. It presents several methodes for measuring radon activity concentration and the potential alpha energy from its short life daughters. It has been prepared by the commission M60-3, of the Office for the standardization of nuclear equipments (BNEN in French) under the French association for standardization (AFNOR in French).

\section{Introduction}

Le radon est un gaz radioactif qui provient de la désintégration de l'uranium et du thorium présents dans la croûte terrestre. Sa désintégration donne naissance à des éléments eux-mêmes radioactifs puis à du plomb stable. Le radon fait partie de la classification chimique des gaz rares comme le néon, le krypton, le xénon,...

1. Institut de Protection et de Sûreté Nucléaire - bâtiment 389 - 91191 Gif-Sur-Yvette Cedex, France

2. FBFC/Romans ; 3. MGP ; 4. EDF ; 5. ALGADE ; 6. Université de Franche Comté ; 7. IPSN/DPHD ;

8. Université de Brest 
Pour l'homme, il est aujourd'hui considéré comme la source principale d'exposition aux rayonnements naturels. D'après le rapport UNSCEAR (1988) il représente en moyenne $37 \%$ environ du bilan radiologique annuel global. La part de l'isotope $222(32 \%)$ est prépondérante sur' l'isotope $220(5 \%)$, (celle du ${ }^{219} \mathrm{Rn}$ est négligeable). Cependant la composante d'exposition due au radon est très variable selon les lieux car elle dépend tout d'abord de la quantité de radon émise par le sol en ces lieux et ensuite du degré de confinement du bâtiment dans lequel se trouve l'individu exposé. On peut ainsi observer des'variations de concentrations d'un facteur 10 ou même d'un facteur 100 d'un moment à un autre et d'un lieu à l'autre.

On trouvera ci-après quelques généralités sur la formation du radon et de ses descendants et les définitions des grandeurs à mesurer, ainsi qu'un descriptif succinct des principáles méthodes de mesure du radon.

\section{Objet et domaine d'application}

On veut pouvoir déterminer la teneur en radon gaz ou/et déscendants dans l'atmosphère libre et l'atmosphère confinée (habitation, local recevant du public, lieu de travail).

Il faut adapter la technique de mesure et sa durée en fonction :

- du but recherché (observation phénoménologique, mesure à caractère sanitaire),

- des niveaux d'activité attendus.

\section{Références nórmatives}

\subsection{Atmosphères intérieures}

Nous reprenons les publications 39, 60 et 65 de la Commission internationale de protection contre les rayonnements. La commission recommande l'utilisation de niveaux d'action, propres à chaque pays, pour la mise en place de techniques de réduction dans les maisons existantes.

Recommandations de la commission des communautés européennes du 21 février 1990 concernant la concentration moyenne annuelle de radon gaz dans les habitations :

\section{La Commission des communautés européennes (...) recommande :}

- qu'un système approprié de réduction de toute exposition aux concentrations de radon à l'intérieur des bâtiments soit établi ; qu'au sein de ce système, l'information adéquate de la population et la réponse à ses projets fassent l'objet d'une attention particulière ; 
- en ce qui concerne les bâtiments existants :

a) que soit fixé un niveau de référence au-delà duquel des mesures simples mais efficaces d'abaịsement du niveau de radon seront envisagées,

b) que ce niveau de référence corresponde à un équivalent de dose efficace de $20 \mathrm{mSv}$ par an, lequel peut être considéré, dans la pratique, comme équivalent à une concentration annuelle moyenne de radon de $400 \mathrm{~Bq} \mathrm{~m}^{-3}(\ldots)$.

- en ce qui concerne les constructions futures :

a) que soit fixé un niveau de conception destiné à guider les autorités compétentes dans l'établissement de règlements de normes ou de codes de pratique de la construction applicables aux cas présentant un risque de dépassement de ce niveau ;

b) que le niveau de conception corresponde à un équivalent de dose efficace de $10 \mathrm{mSv}$ par an, lequel peut être considéré, dans la pratique, comme équivalent à une concentration annuelle moyenne de radon de $200 \mathrm{~Bq} \mathrm{~m}^{-3}(\ldots)$.

\subsection{Environnement des exploitations extractives de substances naturelles radioactives}

\section{- Journal officiel}

Décret $\mathrm{n}^{\circ}$ 90-222 du 9 mars 1990, complétant le règlement général des industries extractives institué par le décret $n^{\circ}$ 80-331 du 7 mai 1980 et concernant la protection de l'environnement. Il faut entendre par :

- exposition naturelle : l'exposition due aux rayons cosmiques et à la présence de substances naturelles radioactives, observable sur le site d'exploitation de telles substances et dans son voisinage, avant le début des travaux,

- exposition ajoutée : la différence entre l'exposition mesurée lorsque l'exploitation de substances radioactives est en activité et l'exposition naturelle.

La limite annuelle des expositions ajoutées est de $2 \mathrm{~mJ}$ d'énergie alpha potentielle pour les descendants à vie courte du radon 222 inhalés,

Remarque : Cette limite est égale à $6 \mathrm{~mJ}$ d'énergie alpha potentielle pour les descendants à vie courte du radon 220 inhalés.

\section{Définitions}

\subsection{Les isotopes du radon}

Le rạdon possède trois isotopes naturels issus des radioéléments présents dans le sol.

Leur abondance respective est, par conséquent, fonction de la nature du sous-sol (teneurs en ${ }^{235} \mathrm{U},{ }^{238} \mathrm{U}$ et ${ }^{232} \mathrm{Th}$ ), mais également de leur période radioactive. 
- Le radon 219 (actinon) est le moins abondant des trois isotopes. En effet, la teneur en $235 \mathrm{U}$ dans les roches et les sols représente environ $0,73 \%$ de celle de l'238U. Par suite, il est pratiquement absent de l'atmosphère et des eaux souterraines. On ne parlera donc, dans ce qui suit, que des deux autres isotopes.

- Le radon 220 (thoron), issu du ${ }^{232} \mathrm{Th}$, est le plus abondant des trois isotopes relâchés par la terre. Cependant, il disparaît très vite de l'atmosphère en raison de sa courte période (55 secondes).

- Le radon 222 , descendant du ${ }^{226} \mathrm{Ra}$, appartenant à la famille de $\mathrm{l}^{238} \mathrm{U}$, émane en quantité plus faible que le thoron (100 fois moins en moyenne). Il est pourtant l'isotope le plus présent dans l'atmosphère à cause de sa période radioactive qui est de 3,8 jours. Cette période est suffisamment longue pour lui permettre de migrer dans les sols depuis la roche qui lui a donné naissance, jusqu'à l'air libre.

Les quantités de ces radionucléides mises en jeu étant très faibles, il est commode de les caractériser par le nombre d'atomes de chacun d'eux, mais c'est généralement leur activité qui est mesurée.

La relation qui lie le nombre d'atomes $N$ et l'activité $A$ s'écrit : $A=\lambda N$

La constante de décroissance $\lambda$ est reliée à la période par la relation :

$$
\lambda=\frac{\ln 2}{T}
$$

Pour l'isotope $222, \lambda$ vaut $2,110^{-6} \mathrm{~s}^{-1}$.

Une activité $A$ de un becquerel (1 Bq), soit une désintégration par seconde, correspond à 476000 atomes de ${ }^{222} \mathrm{Rn}$.

\subsection{L'évolution des concentrations en radon (Robé et al., 1992 ; Wilkening, 1990)}

\subsubsection{Dans les sols}

Le radon trouve son origine principale dans les sols où il est formé par la désintégration des atomes de radium présents dans les minéraux constitutifs des roches. La quantité de ${ }^{222} \mathrm{Rn}$ produit est directement proportionnelle à la teneur du sol en ${ }^{226} \mathrm{Ra}$. Cependant, seule une fraction réussit à s'en échapper, la majorité des atomes produits restant prisonniers du réseau cristallin où ils se désintègrent. Selon la porosité du sol, la taille des grains, l'humidité..., on obtient un taux d'émanation très variable allant de quelques dixièmes de pour-cent à $30 \%$ environ. Une fois échappés du réseau, les atomes de radon sont véhiculés dans l'air ou l'eau du sol, sous l'action de mécanismes de diffusion ou de convection, sur des distances plus ou moins longues. La concentration en ${ }^{222} \mathrm{Rn}$ dans le sol varie dans l'espace (notamment selon un gradient ver- 
tical) et dans le temps, en fonction des conditions météorologiques, des caractéristiques intrinsèques du sol telles que sa perméabilité et sa porosité,...

\subsubsection{A l'interface sol-atmosphère}

La quantité de radon qui arrive à l'air libre par unité de temps et par unité de surface (appelée flux d'émission de ${ }^{222} \mathrm{Rn}$ ) dépend de la concentration en radon dans les sols et de nouveau des conditions météorologiques (IAEA, 1992). Ainsi, le flux d'émission augmente généralement avec le taux d'humidité du sol (lorsque celui-ci varie entre 0 et $80 \%$ environ). et diminue avec la pression atmosphérique. Lorsque le sol est recouvert de neige, d'une lame d'eau ou bien gelé, le flux peut devenir très faible.

Le flux est donc un paramètre explicatif très utile car il intègre à la fois les caractéristiques du sol et les conditions météorologiques (Wilkening, 1990).

\subsubsection{Dans l'atmosphère}

Une fois dans l'air extérieur, le radon se dilue en fonction des conditions de diffusion atmosphérique liées à la météorologie et à l'orographie (Robé et al., 1992). On observe généralement un gradient de concentration vertical, et des variations temporelles selon un cycle journalier. Le jour, la diffusion atmosphérique est le plus souvent bonne ; les concentrations en radon sont relativement faibles. La nuit, on observe fréquemment des inversions de température : la diffusion atmosphérique est très mauvaise, le radon stagne au niveau du sol et sa concentration peut ainsi augmenter dans l'air d'un facteur 10 à 100 (Fig. 1).

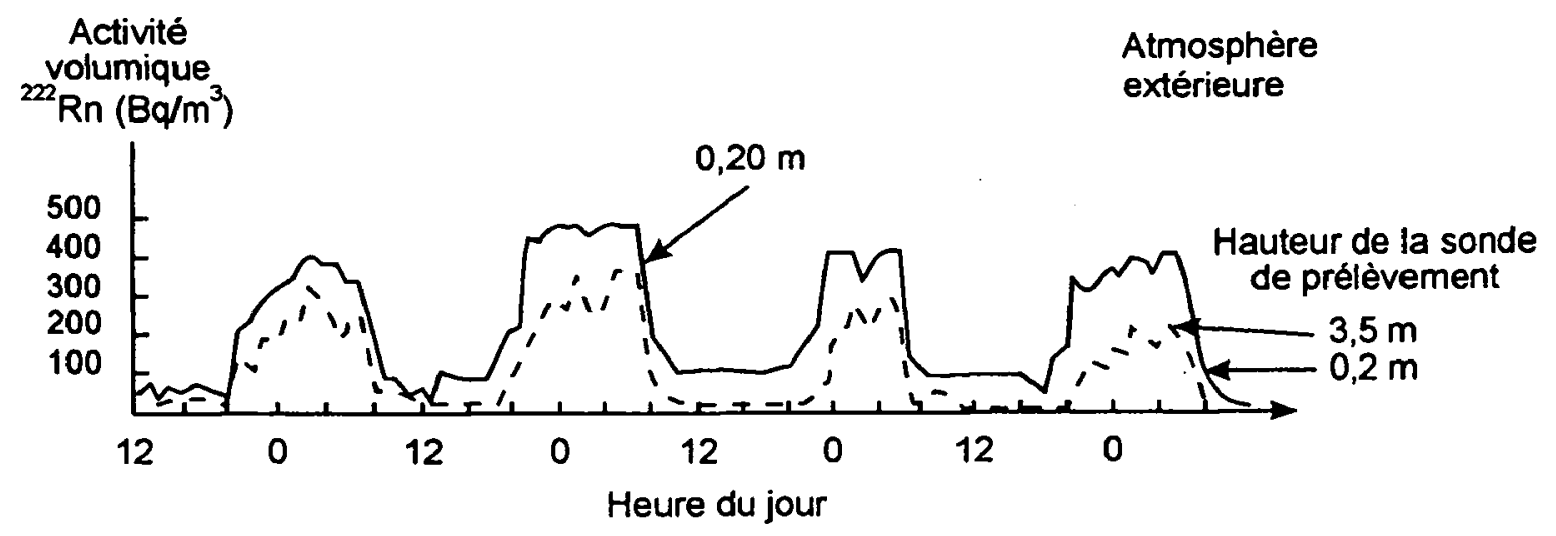

Fig. 1 - Exemple d'évolution des concentrations en radon dans l'atmosphère extérieure (ICRP, Publ. 65).

Example of the developement radon concentration in the outside air

\subsubsection{Dans les maisons}

Dans les maisons, le radon trouve son origine principale dans le sol sousjacent et parfois les matériaux de construction. Les teneurs en radon dépendent également des diverses caractéristiques de l'habitat : 
- nature du soubassement (vide sanitaire, cave, présence de sol en terre battue,...),

- présence ou non d'étages,

- voies de transfert existant entre les différents niveaux (passage de canalisation, escalier,...),

- degré de ventilation et habitudes de vie des occupants.

Plus rarement, l'eau du robinet peut être riche en radon (par exemple lorsqu'elle provient d'un puits situé en terrain granitique) et son dégazage constitue alors une source significative. Selon les régions, l'air extérieur est également une source à considérer.

Le radon se trouve en concentration généralement plus importante dans les maisons que dans l'atmosphère extérieure en raison des plus faibles temps de renouvellement de l'air qui y règnent. A l'inverse de l'atmosphère libre où le radon se dilue puis est transporté à de hautes altitudes, ce gaz s'accumule dans l'air des bâtiments et d'autant plus que la ventilation est réduite (Fig. 2). On observe parfois un cycle journalier de variation de concentrations.

Le processus d'exposition au radon est présenté figure 3.

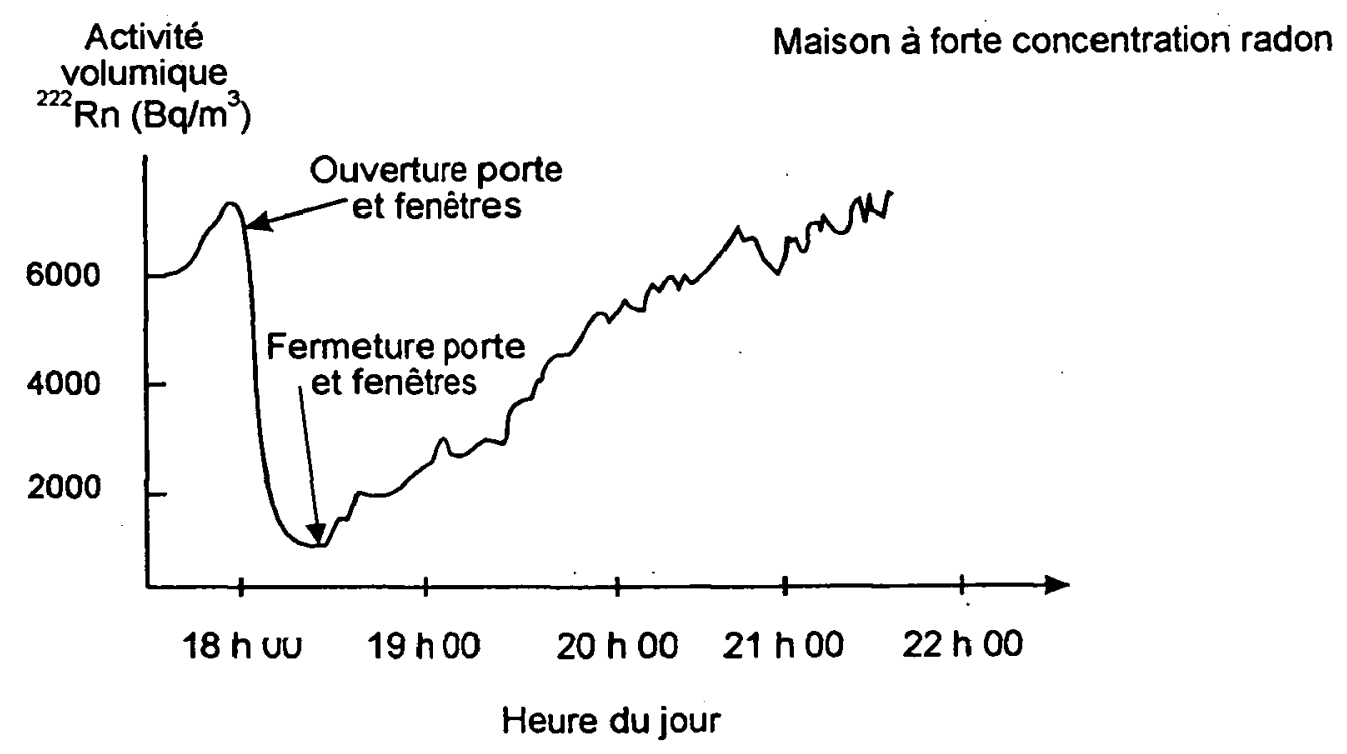

Fig. 2 - Exemple d'évolution des concentrations en radon dans une maison : mise en évidence de l'effet d'une ventilation naturelle (Guélin, 1993).

Example of the development of radon concentration indoors : showing the effects of natural ventilation 


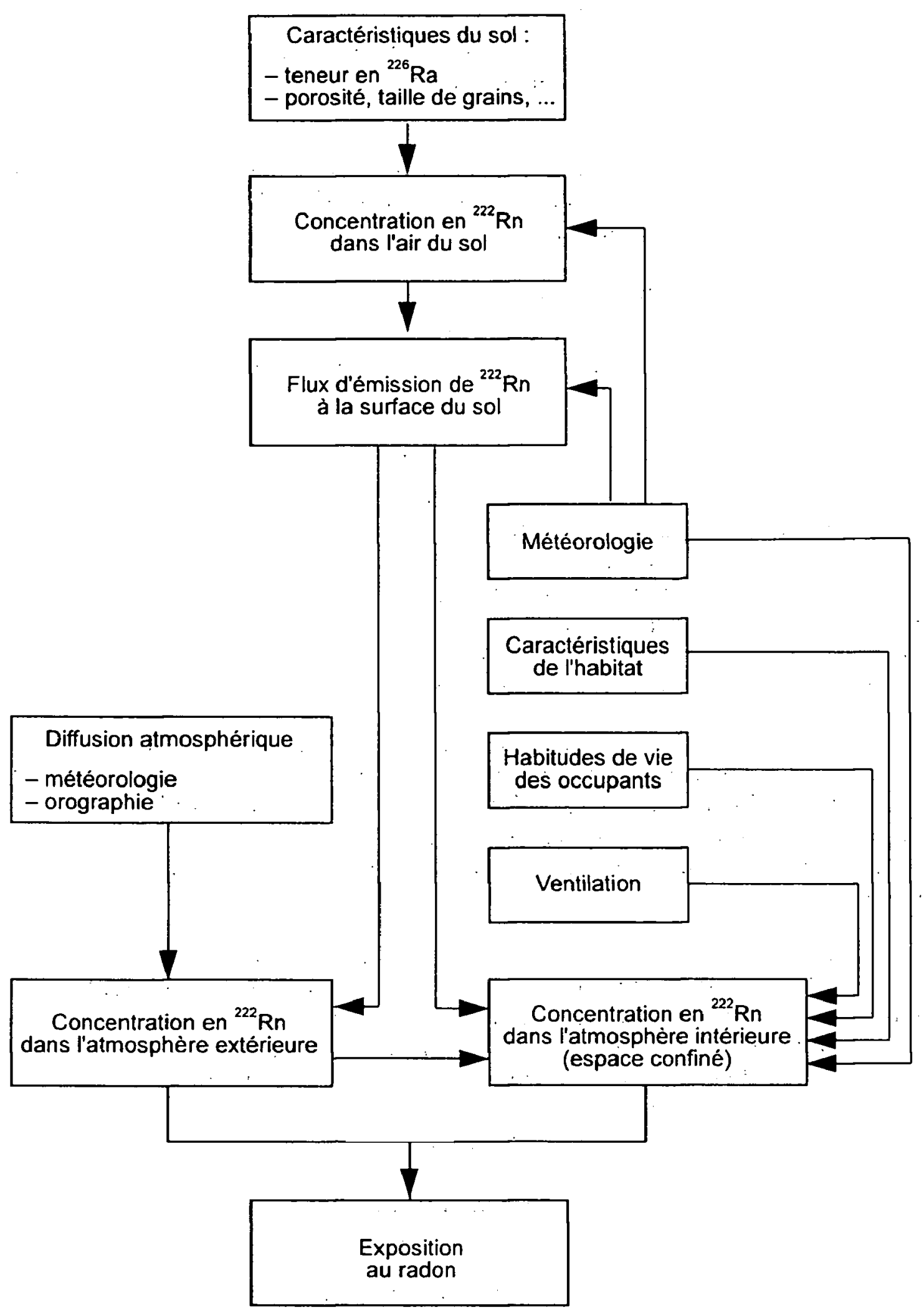

Fig. 3 - Schéma général du processus d'exposition au radon. General diagram of the radon exposure process 


\subsection{Les descendants du radon 222}

En se désintégrant, le radon émet des particules alpha et engendre ainsi des descendants eux-mêmes radioactifs (polonium, bismuth, plomb,...).

Le risque sanitaire du radon n'est pas lié au gaz lui-même, mais à ses produits de filiation. Fixés ou non sur les aérosols atmosphériques, les descendants du radon peuvent alors être inhalés et se déposer dans l'arbre broncho-pulmonaire, plus ou moins profondément, selon leur taille.

\subsubsection{Energie alpha potentielle}

L'énergie alpha potentielle d'un atome est l'énergie alpha totale émise par les désintégrations alpha de cet atome et de ses descendants jusqu'au ${ }^{210} \mathrm{~Pb}$ non inclus.

\subsubsection{Concentration en énergie alpha potentielle}

La concentration en Energie Alpha Potentielle (EAP) due aux descendants à vie courte du radon est définie comme la somme des énergies des particules des descendants du radon qui sont émises lorsque tous les produits de filiation à vie courte (quelles que soient leurs proportions) contenus à un instant donné dans un certain volume d'air pris comme unité, se sont désintégrés (USPHS, 1957). Elle s'exprime généralement en Joule par mètre cube $\left(\mathrm{J} \mathrm{m}^{-3}\right)$ mais aussi en Méga électron-Volt par mètre cube $\left(\mathrm{MeV} \mathrm{m}^{-3}\right)$.

Le radon qui s'est dégagé de la roche se désintègre dans l'air pour donner successivement des atomes de polonium 218, de plomb 214, de bismuth 214 et de polonium 214 (Fig. 4), qui sont donc présents dans l'atmosphère, sous forme de particules submicrométriques. Le descendant suivant, le plomb 210 , de période beaucoup plus longue ( 22 ans), ne s'y trouve qu'en concentration d'activité négligeable.

Ces quatre descendants peuvent être inhalés. Du fait, de sa très courte période $\left(1,610^{-4} \mathrm{~s}\right)$, le polonium 214 ne parvient pas directement dans le poumon. Les trois autres descendants vont s'y fixer, et chacun s'y désintègre jusqu'à donner le plomb 210. Un atome de polonium 218 libère une particule d'énergie alpha de $6 \mathrm{MeV}$ en devenant du plomb 214, qui deviendra ensuite du bismuth 214, puis du polonium 214 qui libère encore une particule alpha d'énergie 7,69 $\mathrm{MeV}$ pour aboutir au plomb 210. La somme de ces deux énergies alpha (soit $13,69 \mathrm{MeV}$ ) est l'énergie alpha potentielle de l'atome de polonium 218. L'énergie alpha potentielle de chaque atome de plomb 214 et de bismuth 214 inhalé n'est que de 7,69 MeV (Tab. I).

La valeur des énergies alpha potentielles pondérées par le nombre d'atomes de chaque descendant mesurés par unité de volume représente l'énergie alpha potentielle volumique. 


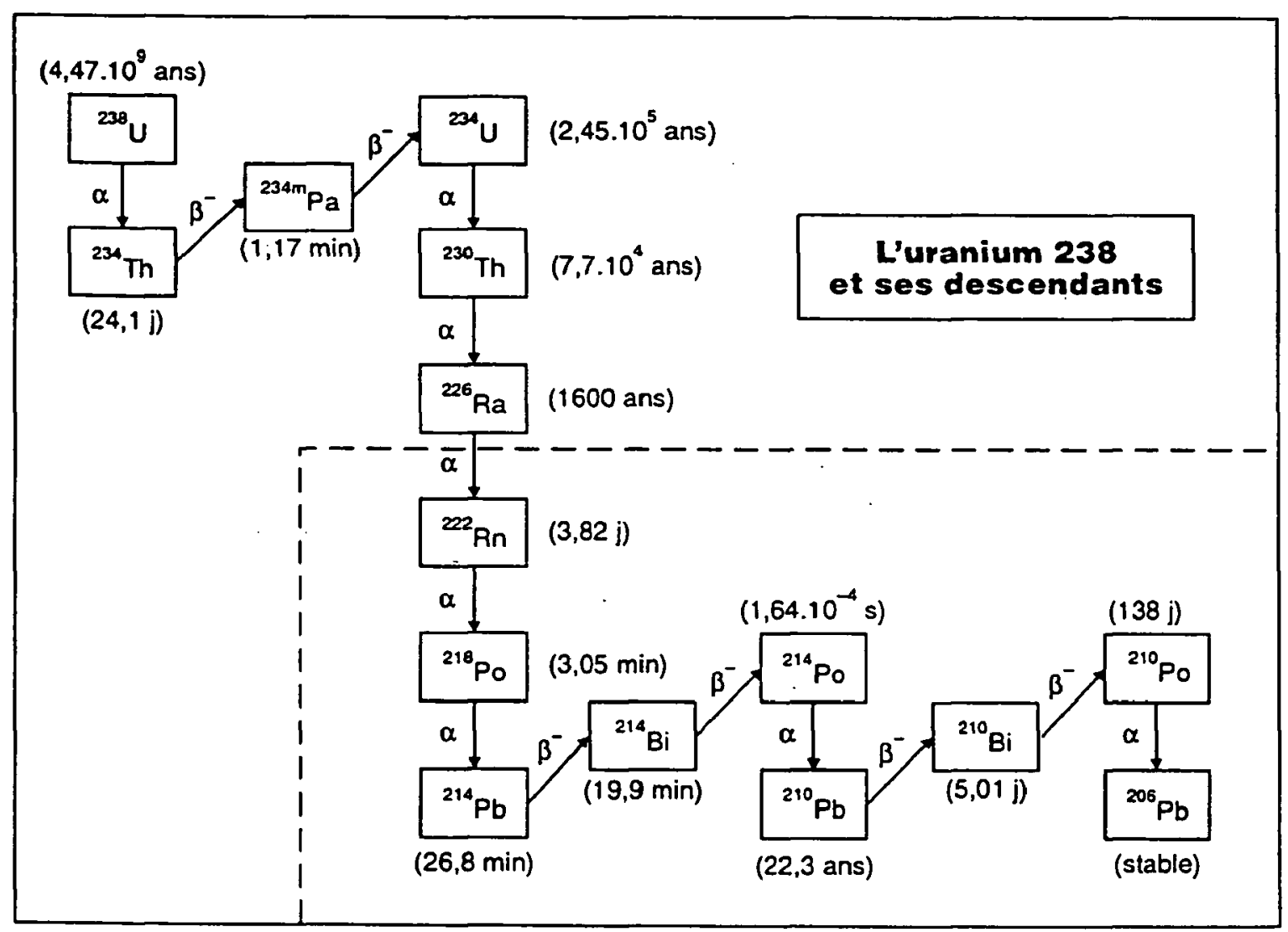

Fig. 4 - Famille radioactive de l'uranium 238 (Rollé, 1972).

Uranium 238 radioactive series

Globalement, l'énergie alpha potentielle volumique $\left(E A P_{\mathrm{V}}\right)$ est égale à :

$$
\begin{aligned}
& E A P_{\mathrm{V}}\left(\text { en } \mathrm{J} \mathrm{m}^{-3}\right)=2,1910^{-12} N^{218} \mathrm{Po}+1,2310^{-12}\left(N^{214} \mathrm{~Pb}+N^{214} \mathrm{Bi}\right) \\
& E A P_{\mathrm{V}}(\text { en } \mathrm{MeV} \mathrm{m}-3)=13,69 N^{218} \mathrm{Po}+7,69\left(N^{214} \mathrm{~Pb}+N^{214} \mathrm{Bi}\right)
\end{aligned}
$$

$N{ }^{218} \mathrm{Po}, N{ }^{214} \mathrm{~Pb}, N{ }^{214} \mathrm{Bi}$ étant respectivement le nombre d'atomes par mètre cube de ${ }^{218} \mathrm{Po},{ }^{214} \mathrm{~Pb}$ et ${ }^{214} \mathrm{Bi}$.

Pour $1 \mathrm{~Bq}$ de radon à l'équilibre avec ses descendants, l'énergie alpha potentielle est égale à $5,5410^{-9} \mathrm{~J}$.

\subsubsection{Facteur d'équilibre}

Les descendants à vie courte du radon 222 présents dans une atmosphère, ne sont jamais à l'équilibre radioactif avec leur père (par suite de leur piégeage sur les parois ou leur élimination grâce à un système de ventilation par exemple).

Pour qualifier cet état de "déséquilibre", on utilise le facteur d'équilibre $F$ que l'on définit comme étant le rapport entre l'énergie alpha potentielle d'un 
mélange de descendants du radon, et l'énergie alpha potentielle relative au mélange s'il était à l'équilibre avec le radon.

$$
F=\frac{E A P_{V}}{5,5410^{-9} C}
$$

$C$ étant l'activité volumique radon mesurée $\left(\mathrm{Bq} \mathrm{m}^{-3}\right), E A P$ la concentration en énergie alpha potentielle volumique mesurée en $\mathrm{J} \mathrm{m}^{-3} ; F$ est un paramètre sans dimension compris entre 0 et 1 .

TABLEAU I

Energie alpha potentielle des descendants à vie courte du 222Rn

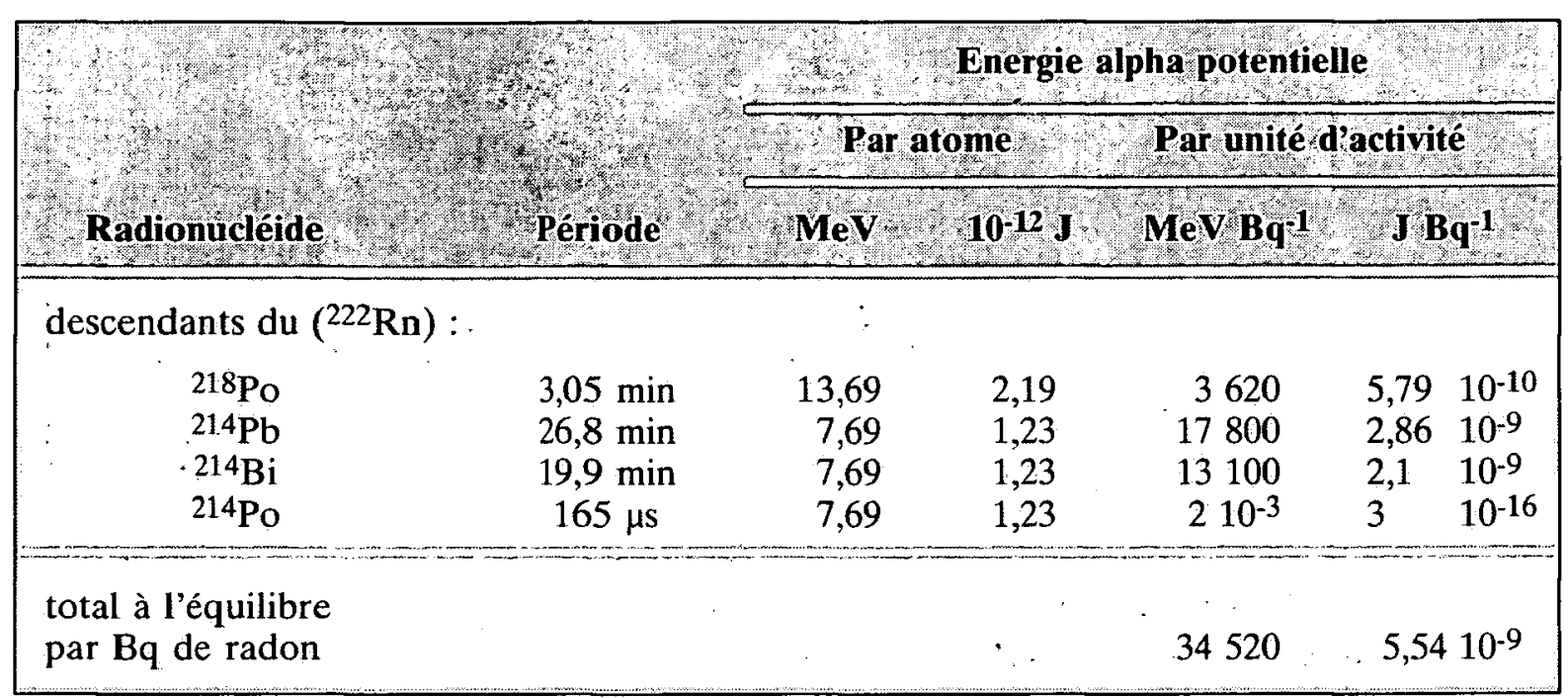

\subsubsection{Concentration en équivalent radon à l'équilibre}

La concentration en équivalent radon à l'équilibre est définie comme étant la concentration en ${ }^{222} \mathrm{Rn}$ d'un mélange, où les descendants seraient en état d'équilibre et auraient la même concentration en énergie alpha potentielle que l'atmosphère réelle étudiée.

Avec les précédentes notations et unités la concentration en équivalent radon à l'équilibre $C_{\text {eq }}$ s'écrit :

$$
\begin{gathered}
C_{\mathrm{eq}}=F C \\
C_{\mathrm{eq}}=\frac{E P A}{5,5410^{-9}}
\end{gathered}
$$

ou :

$C_{\text {eq }}$ s'exprime en $\mathrm{Bq} \mathrm{m}^{-3}$

\subsubsection{Fraction libre et fraction attachée}

Les descendants solides du radon. se présentent sous la forme "libre" ou "attachée". La fraction libre est constituée de particules ultrafines de diamètre 
compris entre $10^{-4} \mu \mathrm{m}$ et quelques $10^{-3} \mu \mathrm{m}$ surtout sous forme de $218 \mathrm{Po}$. Ce sont les descendants sous forme d'ions primaires, secondaires ou de particules neutres. La fraction attachée est constituée d'atomes de descendants libres du radon qui, par diffusion brownienne, se sont attachés aux aérosols ambiants. Sa composante dimensionnelle s'étend de quelques $10^{-2} \mu \mathrm{m}$ à quelques $10^{-1} \mu \mathrm{m}$.

\subsection{Choix du radionucléide à mesurer}

La mesure du radon gaz ne permet pas directement d'accéder au risque sanitaire. L'estimation de ce dernier, à partir de la seule mesure radon gaz, peut se faire au moyen d'un modèle dosimétrique ou en tenant compte du facteur d'équilibre. Dans les habitations le facteur d'équilibre moyen varie entre 0,3 et 0,6 ; en l'absence de valeur exacte, il est communément admis comme égal à 0,4 .

Pour les études portant sur le risque sanitaire, il est plus judicieux de faire une mesure directe des concentrations des descendants sur des périodes de l'ordre de plusieurs semaines. Pour les études à caractère phénoménologique, on peut conseiller la détermination de la concentration en gaz et en descendants.

\subsection{Les unités}

En système SI :

- activité volumique radon gaz : $\mathrm{Bq} \mathrm{m}^{-3}$

- concentration en énergie alpha potentielle volumique : $\mathrm{J} \mathrm{m}^{-3}$.

\section{Mode de prélèvement}

Les grandeurs à mesurer sont généralement des activités volumiques en radon 222 et des concentrations en énergie alpha potentielle volumique. Il existe de nombreuses techniques pour mesurer la concentration de l'air en radon et en descendants du radon.

Elles peuvent être classées en trois catégories selon les caractéristiques du prélèvement d'air ; celui-ci peut être actif ou passif (ni pompe, ni alimentation électrique).

a) Les techniques de mesures ponctuelles consistent en un prélèvement effectué sur une courte période (de l'ordre de quelques minutes ou moins). Le comptage est effectué rapidement par la suite.

b) Dans les techniques de mesure en continu, l'échantillonnage est effectué de façon continue. L'analyse simultanée, ou en léger différé, permet d'enregistrer les variations temporelles de la concentration. 
c) Les techniques donnant des mesures intégrées consistent en un prélèvement effectué sur une longue période (de quelques jours à une année). La mesure en différé fournit, dans ce cas, une valeur globalement représentative de la concentration pendant la période considérée.

Cette classification est commode, même si les limites en sont quelquefois mal fixées, tant pour les mesures de radon que pour celles des descendants.

Pour toute mesure de radon dans l'environnement ou dans une atmosphère confinée il est nécessaire de préciser la durée et la période de mesure. En effet, les activités en radon présentant une très grande variabilité; si la période d'échantillonnage est de quelques minutes, heures ou de plusieurs mois, les résultats obtenus seront très différents entre eux (Fig. 5) et non comparables. La mesure doit se faire dans un lieu dont la situation par rapport aux reliefs naturels ou artificiels voisins place ceux-ci à l'extérieur d'un cône d'axe vertical et d'angle au sommet $140^{\circ}$ au moins, dont le point de prélèvement constitue le sommet. La mesure doit s'effectuer à une hauteur comprise entre 1 et $2 \mathrm{~m} \mathrm{du}$ sol. La hauteur optimale est de 1,50 m (fiche CIRP).
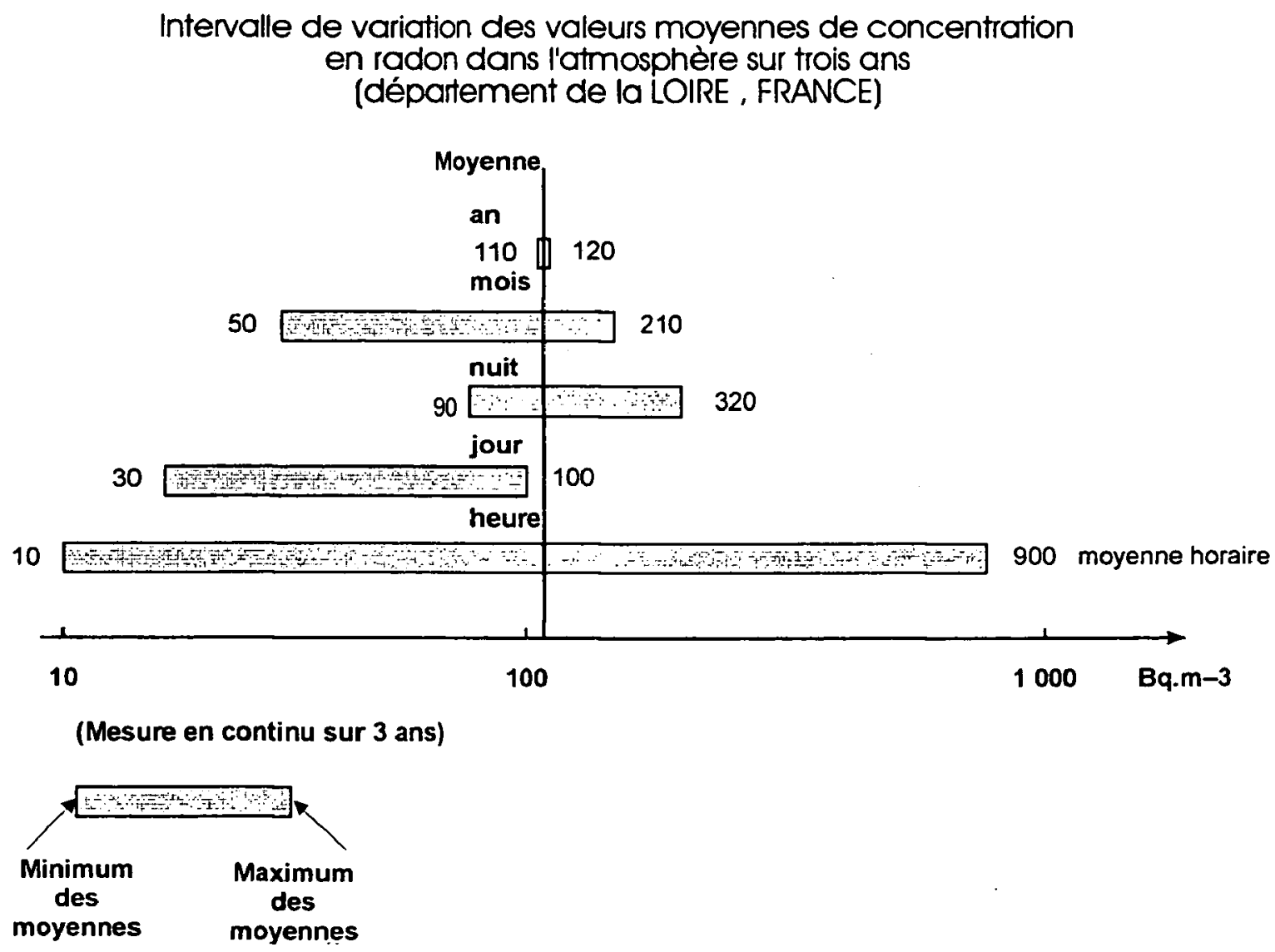

Fig. 5 - Influence de la période d'intégration (ICPR, Publ. 6). Influence of sampling time 


\section{Principe de mesure}

La mesure repose sur différentes méthodes de détection des rayonnements dont l'émission accompagne les désintégrations radioactives successives des isotopes du radon et de leurs descendants. On trouvera ci-après un descriptif succinct de méthodes de mesures reposant sur différents principes (CrawfordBrown et Michel, 1987).

\subsection{Mesure ponctuelle}

\subsubsection{La scintillation du sulfure de zinc activé à l'argent [ZnS (Ag)]}

Le parcours des alpha dans la matière étant très faible (quelques dizaines de micromètres), la particule cède donc toute son énergie au milieu scintillant; cette énergie se retrouve sous la forme de photons qui peuvent être détectés à l'aide d'un photomultiplicateur. C'est le principe adopté pour les fioles scintillantes (par exemple, fioles de Lucas) utilisées pour la mesure du radon gaz.

La scintillation du ZnS (Ag) peut aussi être utilisée pour la détection des descendants du radon collectés sur un filtre (méthode de Thomas (1972), méthode de Rolle (1972),...).

\subsubsection{La spectrométrie gamma}

Un conteneur comme celui utilisé pour la mesure de gaz radioactif, est tapissé intérieurement de plusieurs micro-grilles plissées créant ainsi des milliers de petites alvéoles qui jouent le rôle de piège pour les produits de filiation du radon. La quantité totale de radon présente dans le conteneur alvéolé est déterminée à partir de la mesure des descendants $\left({ }^{214} \mathrm{Bi}\right.$ et $\left.{ }^{214} \mathrm{~Pb}\right)$ par spectrométrie gamma (Guélin, 1993).

\subsection{Mesure en continu}

\subsubsection{L'ionisation de l'air}

Chaque particule alpha créera, lors de son parcours dans l'air, plusieurs dizaines de milliers de paires d'ions qui, sous certaines conditions expérimentales, produiront un courant d'ionisation. Ce courant, bien que très faible, est mesurable et la chambre d'ionisation ainsi constituée permet la mesure de l'activité volumique du radon et de ses descendants. Si l'air mis en circulation à travers la chambre est filtré, le signal est proportionnel à l'activité volumique du radon gaz.

\subsubsection{La spectrométrie alpha}

La spectrométrie alpha utilise un détecteur semi-conducteur, par exemple un silicium qui délivre des impulsions d'amplitude proportionnelle à l'énergie des particules alpha incidentes. 


\subsection{Mesure intégrée}

\subsubsection{La thermoluminescence}

Les matériaux thermoluminescents comme le sulfate de calcium, gardent une "trace latente" du passage d'un rayonnement ionisant. Un chauffage ultérieur entraîne une recombinaison ionique accompagnée d'une émission de photons proportionnelle à la dose délivrée au matériau.

Dans la pratique, on utilise un piège à radon (par exemple : le charbon actif) ou à descendants (par exemple un filtre) et deux dosimètres thermoluminescents. Le premier dosimètre placé en regard du piège enregistre les rayonnements gamma ambiants et ceux émis par les descendants du radon. Le second enregistre seulement les rayonnements gamma ambiants. La différence entre les mesures obtenues par les deux dosimètres permet de déterminer la concentration en radon.

\subsubsection{La spectrométrie gamma}

Le radon peut être piégé sur un matériau adsorbant (par exemple : le charbon actif) après une certaine période d'exposition, la quantité totale de radon présente dans le matériau est calculée à partir de la mesure de ses descendants par spectrométrie gamma.

\subsubsection{Les détecteurs solides de traces}

Une particule alpha traversant certains films plastiques (par exemple le nitrate de cellulose) provoque sur son passage une ionisation. Les recombinaisons ioniques après le passage de la particule ne sont pas complètes. Une attaque chimique appropriée sert de révélateur et le film présente des traces sous la forme de trous ou de cônes d'attaque dont le nombre est égal, en première approximation, au nombre de particules alpha ayant pénétré dans le détecteur.

\subsubsection{Décharge d'une surface polarisée à l'intérieur d'une chambre d'ionisation}

Un disque de téflon porté à un potentiel négatif de l'ordre de $100 \mathrm{~V}$, est inséré dans une chambre d'ionisation à électrets en matériau plastique conducteur, de volume déterminé. Le champ électrostatique ainsi créé à l'intérieur de la chambre permet de collecter sur ce disque les ions formés lors de la désintégration du radon et descendants. Par suite de cette collecte d'ions, le potentiel du disque décroît en fonction de la concentration en radon. Un électromètre permet de mesurer cette différence de potentiel qui est directement proportionnelle à la concentration de radon observée sur la période d'exposition.

Le tableau II récapitule les principales méthodes de détection du radon ou de ses descendants à vie courte utilisées au jour de l'établissement de la norme. 
TABLEAU II

Récapitulatif des principales méthodes de mesure du radon et de ses descendants

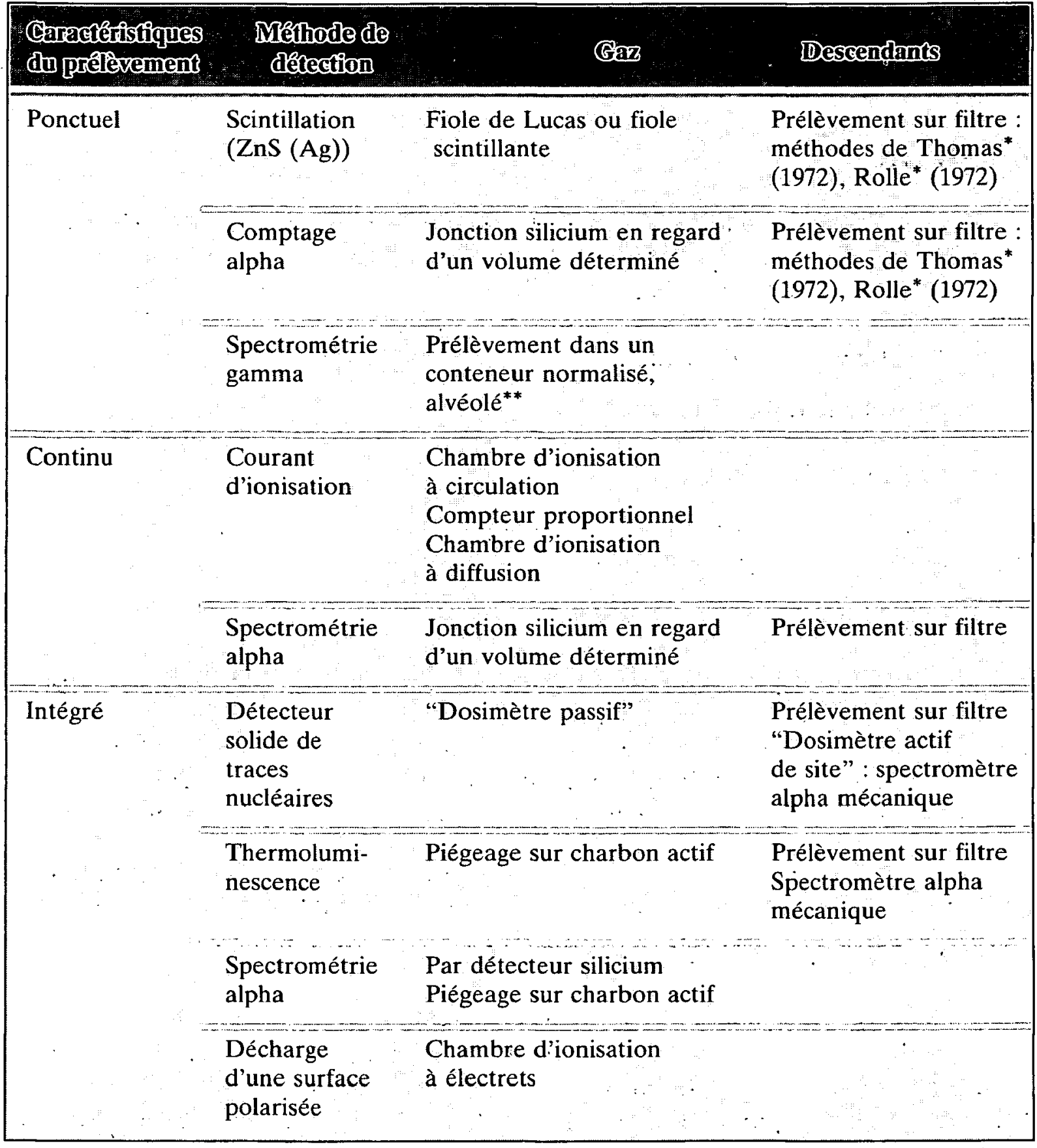

* Méthodes de mesure basées sur la décroissance des descendants à vie courte du radon.

** Cette méthode peut être considérée comme une méthode étalon secondaire (cf. § 5.1.2).

\subsection{Etalonnage des appareils de mesure}

Les différentes méthodes font l'objet d'intercomparaison au niveau international. Il existe également des installations générant des atmosphères contrô- 
lées en radon et descendants. Des prélèvements ponctuels de ces atmosphères peuvent être effectués dans des conteneurs de gaz radioactifs alvéolés qui font l'objet, a posteriori, d'une spectrométrie gamma. Ainsi, ce procédé permet de relier l'activité volumique radon à celles d'étalons primaires gazeux. Ces derniers permettent l'étalonnage de la chaîne de mesure spectrométrique gamma.

\section{Mesure}

Il doit y avoir adéquation entre le phénomène à étudier, le principe de mesure et le mode de prélèvement. Les techniques de mesure du radon et des descendants du radon qui peuvent être utilisées dépendent en grande partie des domaines d'applications (mines, environnement, habitations, locaux recevant du public, lieux de travail,...) et des objectifs visés.

Il existe trois types d'études :

\section{a) Etudes de laboratoire}

Ces études mettent le plus généralement en jeu des méthodes actives (étalonnage d'appareils, étude de sources radifères,...).

\section{b) Etudes à caractère phénoménologique}

Ces études ont pour objet le suivi des variations du radon dans le temps et l'espace en fonction des différents paramètres environnementaux. Cela concerne principalement des études de terrain (mines, atmosphère libre ou diagnostic radon dans les habitations). Les techniques le plus souvent employées sont de type actif (nécessitant un prélèvement d'air au moyen d'une pompe).

Ces deux types d'études (laboratoire et phénoménologique) impliquent la mise en place de matériels lourds, coûteux et nécessitant un personnel qualifié.

\section{c) Etudes à caractère sanitaire}

L'exposition du public au radon est principalement liée à l'habitat et au lieu de travail. Selon l'objectif de l'étude (le dépistage rapide des situations de concentration élevée, l'estimation des niveaux d'exposition), les méthodes de type actif et passif peuvent être utilisées. En général, pour des raisons de coût et de mise en oeuvre, on est conduit à n'utiliser que des méthodes passives (ne nécessitant ni pompe ni alimentation électrique) sur des périodes d'environ 2 mois.

En épidémiologie, où le risque sanitaire est étudié en fonction d'une exposition cumulée sur 20 à 40 ans, une mesure sur 6 mois ou 1 an est préconisée dans l'habitat pour une estimation la plus précise possible de l'exposition individuelle au radon. 
Le résultat de la mesure de la concentration en radon gaz ou en descendants en un lieu donné n'a de signification que s'il est accompagné :

- des modes de prélèvement (ponctuel, intégré, ou continu),

- de la durée du prélèvement,

- de l'époque de la mesure dans l'année,

- des conditions météorologiques.

\section{Conclusion}

En fonction des objectifs visés, il apparaît que les méthodes de mesure à mettre en œuvre sont différentes ainsi que les modes de prélèvement dans l'atmosphère.

Cette description générale des méthodes de mesure du radon 222 et de ses descendants à vie courte dans l'atmosphère sera complétée dans un premier temps par un ensemble de descriptifs précis des méthodes de mesure suivantes :

- mesure intégrée de la concentration en énergie alpha potentielle des descendants à vie courte du radon (par exemple, dosimètre actif de site),

- mesure ponctuelle de la concentration en énergie alpha potentielle des descendants à vie courte du radon (par exemple, méthodes de Thomas et de Rolle),

- mesure en continu de la concentration en radon gaz (par exemple, chambre d'ionisation),

- mesure intégrée de la concentration en radon gaz,

- mesure instantanée de la concentration en radon gaz en atmosphère confinée.

Il conviendra par la suite de s'intéresser aussi à la mesure du flux d'émission radon à l'interface sol-atmosphère.

\section{RÉFÉRENCES}

Crawford-Brown D.J., Michel J. (1987) Measurement in Environmental Radon. Env. Sci. Res. 35 , pp. 59-80 (Cothern C.R., Smith J.E., Eds). Plenum Press: New York and London.

Duchemin B., Coursol N. (1992) Périodes radioactives. Tables de valeurs recommandées CEA/DTA/DAMRI/LPRI, BNM.

Fiche de prélèvement CIRP, GTI 4, Prélèvement sur filtre des matières particulaires en suspension dans l'air ambiant en vue de la détermination de leur radioactivité.

Guélin M. (1993) Réalisatiọn d'un système de référence pour la génération du radon 222. Rapport CEA-R-5636. 
International Agency of Atomic Energy (IAEA) (1992) Measurement of radon concentration and flux. In: Measurement and calculation of radon releases from uranium mill tailings. Tech. Rep. Ser. 333, Vienne.

International Commission on Radiological Protection (ICRP) Publication 39, Principles for limiting exposure of the public to natural sources of radiation, Ann. ICRP 14 (1).

International Commission on Radiological Protection (ICRP) (1990), Publication 60, Recommendations of the International Commission on Radiation Protection, Ann. ICRP 21 (1-3).

International Commission on Radiological Protection (ICRP) (1993), Publication 65. Protection Against Radon-222 at Home, Ann. ICRP 23 (2).

Robé M.C., Rannou A., Le Bronec J. (1992) Radon Measurements in the Environment in France. Radiat. Prot. Dosim. 45 (1-4), 455-457.

Robé M.C., Rannou. A., Le Bronec J., Tymen G. (1990) Le radon dans les habitations : identification des voies de transfert, et caractérisation des aérosols radioactifs produits. In: Congrès International sur la Géochimie des Gaz, Mons (Belgique) octobre 1990.

Rolle R. (1972) Rapid Working Level Monitoring, Health Phys. 22, 223-238.

Thomas J.W. (1972) Measurement of Radon Daughters in Air, Health Phys. 23, 783-789.

United States Public Health Service (USPHS) (1957) publication $n^{\circ} 494$.

UNSCEAR (1993) Ionizing Radiation Sources and Biological Effects. 1993 Report to the General Assembly with Annexes. UNSCEAR Publ.: New York.

Wilkening M. (1990) Radon in environment studies. Env. Sci. 40. 